\title{
Low-frequency noise in planar Hall effect bridge sensors
}

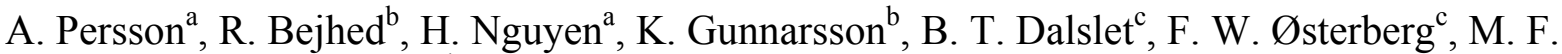 \\ Hansen $^{\mathrm{c}}$, and P. Svedlindh ${ }^{\mathrm{b}}$ \\ ${ }^{a}$ Ångström Space Technology Centre, Uppsala University, Ångström Laboratory Box 534 SE-75121, Uppsala, \\ Sweden \\ ${ }^{b}$ Division of Solid State Physics, Dept. of Engineering Science, Uppsala University, Ångström Laboratory Box \\ 534 SE-75121, Uppsala, Sweden \\ ${ }^{c}$ Dept. of Micro- and Nanotechnology, Technical University of Denmark, DTU Nanotech, Building 345 East, \\ DK-2800 Kongens Lyngby, Denmark
}

\section{Corresponding author}

Anders Persson

Box 534, 75121 Uppsala, Sweden

Phone: +46184713111, Fax: +46184713572, email: anders.persson@angstrom.uu.se

\begin{abstract}
The low-frequency characteristics of planar Hall effect bridge sensors are investigated as function of the sensor bias current and the applied magnetic field. The noise spectra reveal a Johnson-like spectrum at high frequencies, and a $1 / f$-like excess noise spectrum at lower frequencies, with a knee frequency of around $400 \mathrm{~Hz}$. The $1 / f$-like excess noise can be described by the phenomenological Hooge equation with a Hooge parameter of $\gamma_{\mathrm{H}}=0.016$. The detectivity is shown to depend on the total length, width and thickness of the bridge branches. Increasing the total length by a factor of 10 improves the detectivity by a factor of $10^{1 / 2}$. Moreover, the detectivity is shown to depend on the amplitude of the applied magnetic field, revealing a magnetic origin to part of the $1 / f$ noise.
\end{abstract}

Keywords: Magnetoresistance, Planar Hall effect, Low-frequency noise, Detectivity

\section{Abbreviations ${ }^{1}$}

\section{Introduction}

Magnetic field sensors based either on the intrinsic anisotropic magnetoresistance (AMR) effect of ferromagnetic materials, or the giant magnetoresistance (GMR) and tunnelling magnetoresistance (TMR) effect of ferromagnetic/non-magnetic heterostructures are today utilized in many areas of science and technology [1,2]. With reports of magnetoresistance ratios of several hundred percent at room temperature, much work is presently focused on TMR sensors with the ambition to develop a magnetic field sensor with picotesla sensitivity [3-7].

The minimum detectable magnetic field of a sensor is determined by its intrinsic noise. While it has been possible to achieve close to picotesla sensitivity at high frequencies (typically at frequencies higher than some tens of $\mathrm{kHz}$ ), it has been proven more difficult to achieve the sensitivity goal at low frequencies due to $1 / f$ resistance noise. The $1 / f$ noise can have both magnetic origins, due to coupling between transport properties and magnetization fluctuations,

\footnotetext{
${ }^{1}$ AMR - Anisotropic magnetoresistance, GMR - Giant magnetoresistance, TMR - Tunneling magnetoresistance, PHE - Planar Hall effect, PHEB - Planar Hall effect bridge, PSD - Power spectral density, SRS - Standard Research Systems
} 
and electronic origins [8-12]. For TMR devices, the latter contribution is often attributed to defects in the tunnel barrier resulting in electron trapping with thermally activated kinetics and a broad distribution of activation energies, but it should be noted that $1 / f$ noise is also found in metals where carrier scattering by extrinsic defects has been pointed out as the source of the resistivity fluctuations [13]. Attempts to improve field detectivity at low frequencies include low temperature annealing [14, 15] and using flux concentrators to amplify the magnetic field at the sensor $[5,6,16,17]$. An interesting suggestion is to use an ac microelectromechanical flux concentrator system to mitigate the nuisance of $1 / f$ noise by modulating the sensor magnetic field to a higher frequency regime where the $1 / f$ noise is lower [18].

On one hand, the magnetoresistance ratio is much larger for TMR and GMR sensors than for AMR sensors. On the other hand, the excess $1 / f$ noise is in general much smaller for AMR sensors and therefore it may even be that AMR based sensors exhibit better magnetic field detectivity than TMR and GMR sensors at low frequencies [2]. Planar Hall effect (PHE) sensors, which are based on the AMR effect, are presently being explored in substrate based [19] as well as for substrate free magnetic biosensors applications [20]. An interesting development of PHE sensors is to replace the traditional cross-shaped Hall geometry with a Wheatstone bridge design. By carefully choosing the bridge geometry, it has recently been shown that it is possible to enhance the sensor output by a factor of 100 compared to crossshaped sensors [21].

In this article we present results from low-frequency voltage noise measurements as a function of magnetic field and sensor geometry on Wheatstone bridge PHE sensors. The planar Hall effect bridge (PHEB) sensors have the same geometry and nominal structure as those studied in [21], but are from a different fabrication batch. The power spectrum of the noise is frequency independent above $400 \mathrm{~Hz}$ and exhibits a $1 / f$ character at lower frequencies. The noise has its origin in Johnson noise and magnetic as well as non-magnetic $1 / f$ noise sources. The frequency dependent magnetic field detectivity for the PHE sensors has been calculated and it is shown that the field detectivity of PHEB sensors can be at least an order of magnitude lower than that obtained for corresponding cross-shaped PHE sensors.

Figure 1 here.

\section{Experimental}

PHEBs with exchange biased branches were deposited by magnetron sputtering on a thermally oxidised Si wafer [21]. The thin-film structure of the PHEBs was substrate / Ta (5 $\mathrm{nm}) / \mathrm{Ni}_{80} \mathrm{Fe}_{20}(30 \mathrm{~nm}) / \mathrm{Mn}_{76} \mathrm{Ir}_{24}(20 \mathrm{~nm}) / \mathrm{Ta}(5 \mathrm{~nm})$, where numbers in parentheses denote the film thickness and subscripts the concentration in at.\%. Meander PHEBs were patterned using UV-lithography and lift-off with varying length, $l$, number of segments, $n$, each of length $l$, and width, $w$ (Fig. 1(a), (b)). In total, 8 PHEBs were fabricated, from here on referred to as PHEB \#1 to \#8, with specifications according to Table I. During film deposition, a magnetic field of $20 \mathrm{mT}$ was applied along the $x$-axis (Fig. 1(c)) to define the direction of the exchange coupling between the ferromagnetic Ni-Fe layer and the antiferromagnetic Mn-Ir layer, yielding the direction of the magnetization vector in zero field $\hat{\mathbf{m}}(H=0)=(1,0)$.

The PHEBs were wire-bonded to a printed circuit board for magnetoresistance characterization. The $1^{\text {st }}$ harmonic in-phase component of the PHE voltage, $V_{\mathrm{y}}$, was measured by a Stanford Research Systems (SRS) SR830 lock-in amplifier after 40 dB preamplification 
in an SRS552 preamplifier, while applying a magnetic field, $\mu_{0} H_{y}$, along the $y$-axis (Fig. 1(c)). The magnetic field was varied between $\pm 40 \mathrm{mT}$. The PHEBs were biased with an alternating current, $I_{\mathrm{p}-\mathrm{p}} \sin (2 \pi f t)$, with $I_{\mathrm{p}-\mathrm{p}}=1 \mathrm{~mA}$ and $f=2.2 \mathrm{kHz}$ supplied by a Keithley 6221 current source.

The noise of the PHEBs was measured with an HP 35670A spectrum analyzer. The PHEBs were biased with a direct current of $I=10 \mathrm{~mA}$ supplied from lead-acid batteries to minimize the intrinsic noise of the current source. Likewise, the current through the electromagnetic coil was supplied by lead-acid batteries to avoid noise from the power system. Before being sampled by the spectrum analyzer, the PHEB voltage noise was amplified by $40 \mathrm{~dB}$ using an HMS Electronics Model 565 low-noise amplifier. The inherent Johnson noise level of the amplifier was $1.24 \mathrm{nV} / \sqrt{\mathrm{Hz}}$. The noise of the PHEBs was measured in two overlapping frequency regions, $125 \mathrm{mHz}$ to $200 \mathrm{~Hz}$ (or $250 \mathrm{mHz}$ to $400 \mathrm{~Hz}$ ), and $8 \mathrm{~Hz}$ to $12.8 \mathrm{kHz}$. The noise of PHEB \#1 was studied in more detail by varying the bias current, and the magnetic field. A schematic view of the noise measurement system is shown in Fig. 2.

Figure 2 here.

\section{Theory}

The PHE voltage, $V_{\mathrm{y}}$, of a traditional cross-shaped PHE resistor, magnetized in-plane, along the unit vector $\hat{\mathbf{m}}=(\cos \theta, \sin \theta)$, where $\theta$ is the angle between the magnetization vector and the anisotropy axis (Fig. 1 (c)), has been shown to be well described by a single magnetic domain model giving:

$$
V_{y}^{\text {cross }}=I \Delta \rho \sin (2 \theta)\left(2 t_{\mathrm{FM}}\right)^{-1}
$$

where $I$ is the uniform current carried by the resistor with thickness, $t_{\mathrm{FM}}$, and $\Delta \rho=\rho_{\|}-\rho_{\perp}$ is the difference in resistivity with the magnetization parallel and perpendicular to $I$ [21].

The PHE voltage of a meander shaped PHEB, with branches at $\alpha= \pm 45^{\circ}$, where $\alpha$ is the angle between the current vector and the anisotropy axis (Fig. 1 (c)), resembles that of a crossshaped sensor except for the geometric amplification factor, $n l / w$ [21]:

$$
V_{\mathrm{y}}=(n l I \Delta \rho \sin (2 \theta))\left(2 w t_{\mathrm{FM}}\right)^{-1}
$$

For such a PHEB sensor, exchange biased along $\theta=0$, the sensitivity, $\Sigma$, for small magnetic fields, $H_{y}$, at a certain bias current is

$$
\Sigma\left[\mathrm{VT}^{-1}\right]=\mu_{0}^{-1}\left(\partial V_{\mathrm{y}} / \partial H_{\mathrm{y}}\right)=[n l I \Delta \rho] \cdot\left[w t_{\mathrm{FM}}\left(H_{\mathrm{ex}}+H_{\mathrm{K}}\right)\right]^{-1},
$$

where $H_{\mathrm{ex}}$ is the exchange bias field and $H_{\mathrm{K}}$ is the field-induced uniaxial anisotropy of the NiFe layer.

At low frequencies, the most prominent noise sources in a magnetoresistive sensor are Johnson noise and $1 / f$ noise [22]. Johnson noise, which is also called thermal, white, or Nyquist-noise, occurs in all resistors and is caused by random thermal motion of electrons [23]. The power spectral density (PSD), $S_{\mathrm{J}}$, of the Johnson noise is given by 


$$
S_{\mathrm{J}}\left[\mathrm{V}^{2} \mathrm{~Hz}^{-1}\right]=4 k_{\mathrm{B}} T R
$$

where $k_{\mathrm{B}}$ is Boltzmann's constant, $T$ is the temperature and $R$ is the bridge resistance.

$1 / f$ noise in magnetoresistive devices can have both magnetic and electric origin; however, it can be described by the phenomenological Hooge equation [24]

$$
S_{1 / f}\left[\mathrm{~V}^{2} \mathrm{~Hz}^{-1}\right]=V^{2} \gamma_{\mathrm{H}}\left(N_{\mathrm{C}} f\right)^{-1} \text {, }
$$

where $S_{1 / f}$ is the PSD of the $1 / f$ noise, $V=I R$ is the bias voltage, $N_{\mathrm{C}}$ is the number of charge carriers, $f$ is the frequency and $\gamma_{\mathrm{H}}$ is the dimensionless Hooge parameter.

The detectivity, $D_{\mathrm{B}}$, of a magnetoresistive device refers to the lowest detectable magnetic field. It is calculated from the total, inherent voltage noise PSD, $S_{\mathrm{V}}$, and the low field sensitivity and is given by:

$$
D_{\mathrm{B}}\left[\mathrm{THz}^{-1 / 2}\right]=S_{V}^{1 / 2} \Sigma^{-1}=\left(S_{\mathrm{J}}+S_{1 / f}\right)^{1 / 2} \Sigma^{-1},
$$

where $S_{\mathrm{V}}$ is calculated from the recorded spectrum, $S_{0}$, by subtracting the amplifier noise, $S_{\text {Amp: }}$ :

$$
S_{\mathrm{V}}=S_{0}-S_{\mathrm{Amp}}
$$

\section{Results and discussion}

The response of the PHEB designs used in this study has been studied previously [21]. For the present fabrication batch, $\mu_{0} H_{\mathrm{ex}}=3.3 \mathrm{mT}$ and $\mu_{0} H_{\mathrm{K}}=0.4 \mathrm{mT}$ were obtained from easy axis hysteresis loops measured in a vibrating sample magnetometer on a lithographically defined $3 \times 3 \mathrm{~mm}^{2}$ square sample geometry. From electrical measurements on a transmission line structure in a saturating magnetic field applied along and perpendicular to the current direction, $\Delta \rho / t_{\mathrm{FM}}=0.186 \Omega$ was obtained, corresponding to an AMR ratio of $2.3 \%$. The AMR ratio was slightly higher and the values of $\mu_{0} H_{\text {ex }}$ and $\mu_{0} H_{\mathrm{K}}$ were slightly lower than those obtained in [21] resulting in sensitivity values that were typically about $20 \%$ higher than those obtained in [21].

Figure 3 shows the values of $V_{y}$ (corresponding to Eq. (2)) measured for the various studied sensor geometries normalized by the applied current amplitude. Table I lists the sensor geometries and shows the low-field sensitivities obtained from linear fits in the low-field region.

Figure 3 here.

Table 1 here.

To investigate the low-frequency noise of the PHEBs, their noise spectra were recorded while biased with a direct current of $10 \mathrm{~mA}$. The detectivity of each PHEB was then calculated from Eqs. (6) and (7) (Fig. 4). The noise, measured in an interval from $0.125 \mathrm{~Hz}$ to $12.8 \mathrm{kHz}$, 
showed a Johnson-like noise at high frequencies, and $1 / f$-like excess noise at lower frequencies, with a knee frequency of around $400 \mathrm{~Hz}$.

Figure 4 here.

To confirm that the excess $1 / f$-like noise originated from the PHEBs, and not from the noise measurement setup or from the electrical contacts, the noise properties of PHEB \#1 were studied more carefully, varying $I$. If the noise originated from the PHEB, the $1 / f$ part of the PSD would show a $S_{V}^{1 / 2} \sim I$ dependence according to Eq. (5). This was confirmed by the measurements, as can be seen in Fig. 5 . Hence, it was concluded that the $1 / f$-like excess noise seen in the spectra was inherent to the PHEBs.

Figure 5 here.

The voltage $1 / f$-noise has been shown to be inversely proportional to the number of charge carriers in the system and thus, the volume of the sample [24]. This effect could also be seen in the PHEBs, when examining the relationship between the detectivity and the total length of the PHEBs, nl, (which determines the difference in sensor volume between PHEBs \#2-\#8 since $w$ and $t_{\mathrm{FM}}$ were the same for these sensors).

Figure 6 here.

Below the knee frequency, where $S_{1 / f}$ dominates the noise spectrum, $S_{\mathrm{V}}^{1 / 2}$ is expected to follow a $(n l)^{1 / 2}$-dependence $\left(\sim R N_{\mathrm{C}}^{-1 / 2}\right.$, cf. Eq. (5)) and, normalized with $\Sigma(\sim n l$, cf. Eq. (3)), a $(n l)^{-1 / 2}$-dependence is found for the detectivity. In Fig. 6, $D_{\mathrm{B}}$ is plotted as a function of $n l$ and the expected dependence is confirmed. Including $w$ and $t_{\mathrm{FM}}$ in the analysis, and using $S_{V}^{1 / 2} \sim I R\left(n l w t_{\mathrm{FM}}\right)^{-1 / 2}$ (cf. Eq. (5)), $\Sigma \sim I R\left(H_{\mathrm{ex}}+H_{\mathrm{K}}\right)^{-1}$ (cf. Eq. (3)), $I=J w t_{\mathrm{FM}}$ where $J$ is the current density, $R \sim n l\left(w t_{\mathrm{FM}}\right)^{-1}$ and $H_{\mathrm{ex}}+H_{\mathrm{K}} \sim\left(t_{\mathrm{FM}}\right)^{-1}$ [25], yield the relation $D_{\mathrm{B}} \sim\left(n l w t_{\mathrm{FM}}^{3}\right)^{-1 / 2}$, indicating that the detectivity can be further improved by increasing $n l, w$, and/or $t_{\mathrm{FM}}$. Above the knee frequency, where $S_{\mathrm{J}}$ dominates the noise spectrum $S_{\mathrm{V}}^{1 / 2}$ is proportional to $R^{1 / 2}$ (cf. Eq. (4)), whereas the detectivity, again, is proportional to $\left(n l w t_{\mathrm{FM}}^{3}\right)^{-1 / 2}$, assuming $J$ to be constant. Consequently, the knee frequency is independent of the PHEB geometry, since $D_{\mathrm{B}}$ has the same dependence on $n l w t_{\mathrm{FM}}$ in both frequency regimes. Increasing $t_{\mathrm{FM}}$ is the most effective measure to reduce $D_{\mathrm{B}}$, however, $t_{\mathrm{FM}}$ can only be increased as long as the single-domain configuration is maintained - the limit being around $50 \mathrm{~nm}$ [25]. On the other hand, nlw can be increased several orders of magnitude without loosing the single-domain configuration. To validate the dependency of the detectivity on the width, the detectivity of PHEB \#1 at $10 \mathrm{~Hz}$, $D_{\mathrm{B} \# 1}=5.58 \cdot 10^{-9} \mathrm{THz}^{-1 / 2}$, was scaled to a PHEB with $w=30 \mu \mathrm{m}$ and $l=100 \mu \mathrm{m}$ according to the derived model, $D_{\mathrm{B}} \sim(l w)^{-1 / 2}$, showing good agreement with the model prediction (Fig. 6).

Magnetic low-frequency noise, also called Barkhausen noise, stems from fluctuating magnetic domains [26]. The domains can fluctuate both in size and in magnetization direction, and since the magnetoresistive response of a device is dependent on the domain configuration, and the domain magnetization direction, this causes noise in the output signal. Magnetic low- 
frequency noise is strongly associated with conflicting magnetic anisotropies [10,11, 17], for the PHEBs, e.g., in the sharp corners where the easy axis of the shape anisotropy changes direction and, also, in the PHEB branches where the shape anisotropy is partly opposed by the exchange bias and field-induced uniaxial anisotropies. Given the larger number of such corners, a PHEB with $n>1$ could have been expected to have worse detectivity than one with $n=1$ and equal length. However, the detectivity of the PHEBs with $n>1$ (\#6-\#8) showed to be in line with that of the PHEBs with $n=1$ (\#1-\#5) (Fig. 6), confirming that the meander design was a good way of improving the detectivity without drastically increasing the size of the device. The Hooge parameter, $\gamma_{H}$, was calculated from Eq. (5) for each PHEB, giving a mean value of $\gamma_{\mathrm{H}}=0.016$, which is only one order of magnitude higher than typical values of $\gamma_{\mathrm{H}}$ for well crystallized metallic films [22] and lower than for many magnetoresistive sensors [1, 8, $27,28]$. Here, $N_{\mathrm{C}}$ was estimated from the charge carrier density of $\mathrm{Ni}_{80} \mathrm{Fe}_{20}, n_{\mathrm{Ni}-\mathrm{Fe}}=17 \times 10^{28}$ $\mathrm{m}^{-3}$ [29], assuming that the majority of $I$ was conducted though the NiFe layer given that $\rho_{\mathrm{Ni-Fe}}<<\rho_{\mathrm{Mn}-\mathrm{Ir}}<\rho_{\mathrm{Ta}}[30]$.

Three anisotropies influenced the PHEBs: the field-induced uniaxial anisotropy with easy axis at $\theta=\theta_{\mathrm{K}}=0^{\circ}$ and $180^{\circ}$, the exchange bias anisotropy with easy axis at $\theta=\theta_{\mathrm{ex}}=0^{\circ}$, and the shape anisotropy with easy axes at $\theta=\theta_{\mathrm{S}}=\alpha= \pm 45^{\circ}$, (Fig. 1(c)). To study the low-frequency noise at and in-between these anisotropy directions, the detectivity of PHEB \#1 was investigated at different applied magnetic fields (Fig. 7). The magnetic part of the noise is reduced if the magnetization is directed along an anisotropy axis [10,11, 17], and the detectivity should therefore have local minima at $\theta=\theta_{\mathrm{ex}}$ and $\theta_{\mathrm{S}}$. This was confirmed by the measurements. The comparably deep minimum at $\theta=\theta_{\mathrm{S}}$ is an effect of the field derivative of $V_{y}$ being close to zero for this direction of the magnetization. The part of the PHE voltage curve corresponding to the linear regime exhibits the largest noise with a tendency to increase when moving away from $\theta=\theta_{\mathrm{s}}$. However, for $-\theta_{\mathrm{S}}<\theta<\theta_{\mathrm{S}}$ the voltage noise was more or less constant.

\section{Figure 7 here.}

\section{Conclusion}

The low-frequency noise and detectivity of planar Hall effect bridges have been studied experimentally, and the noise has been shown to have a $1 / f$-dependence below $400 \mathrm{~Hz}$. Moreover, the $1 / f$-noise can be described by the phenomenological Hooge equation (Eq. (5)), with a Hooge parameter $\gamma_{\mathrm{H}}=0.016$. The dependence of the detectivity on the geometry of the sensors was also investigated, yielding $D_{\mathrm{B}} \sim(n l)^{-1 / 2}$ in both the high and low-frequency regime. Incorporating the width and thickness of the PHEBs in the analysis yielded $D_{\mathrm{B}} \sim\left(n l w t_{\mathrm{FM}}^{3}\right)^{-1 / 2}$ although this relationship has to be verified experimentally. Increasing $n l$ gave an improvement of the detectivity following $D_{\mathrm{B}}=4 \cdot 0 \cdot 10^{-11}(\mathrm{nl})^{-1 / 2} \mathrm{THz}^{-1 / 2}$. To optimize the lowfrequency performance of the PHEBs, $t_{\mathrm{FM}}$ should be made as thick as possible, maintaining the single-domain configuration, whereas $n l w$ can be varied to fulfil any requirements on the detectivity and the size of the device. Furthermore, the dependence of the detectivity on the applied magnetic field was also studied, showing part of the low-frequency noise to have a magnetic origin. The magnetic part of the $1 / f$-noise was found to be most pronounced in the linear field-range of the sensors.

Comparing the performance of the sensors presented here with that of ordinary cross-shaped planar Hall sensors with the same width $w$ we find experimentally that the detectivity is improved by the square root of the sensitivity improvement. The use of cross-shaped planar 
Hall sensors for in-channel integrated detection of the dynamic magnetic response of magnetic beads has recently been demonstrated [20]. In that work, the oscillating magnetic field magnetizing the magnetic beads was due to the ac bias current applied to the sensor. The increased detectivity of the PHEBs and their larger sampling volume due to their larger size indicate that the use of PHEB sensors for on-chip self-field detection of magnetic beads is very promising. This is a topic of our further studies.

Making an objective comparison between PHEBs and other magnetoresistive sensors is difficult since the response, size and complexity of the different devices differ considerably. Comparing the detectivity of the sensors presented here to commercial AMR, GMR and TMR sensors [2] suggests that the PHEB performance is well in line, or even better than the competition. Given the opportunity of reducing the detectivity of the PHEBs even further, by increasing the dimensions of the bridges, these sensors show great promises for use in future thin-film, low-frequency magnetometers.

\section{Acknowledgements}

Financial support from the Knut and Alice Wallenberg Foundation (KAW), the Swedish Research Council (VR), the Danish Council for Independent Research, Technology and Production Sciences and Copenhagen Graduate School for Nanoscience and Nanotechnology is gratefully acknowledged.

\section{Biographies}

Anders Persson was born in 1982 and received his MSc in engineering physics from Uppsala University, Sweden, in 2007. He is now working as a PhD student at the Ångström Space Technology Centre at Uppsala University, researching magnetoresistive sensors and their applicability to space.

Rebecca Stjernberg Bejhed, born in Uppsala, Sweden 1978. Master of Science in Materials Engineering, Uppsala University, 2002. Started her Ph.D. studies 2008 at the Department of Engineering Sciences, Solid State Physics. Area of research, magnetic sensors for bioapplications.

Hugo Nguyen received the M.Sc. degree in machine engineering in 1992 at Lund Institute of Technology and $\mathrm{PhD}$ degree in engineering physics specializing in microsystems technology in 2006 at Uppsala University, both in Sweden. He has been involved in the design of all nanosatellites proposed by the Ångström Space Technology Centre at Uppsala University and has a special interest in all kinds of interfaces between micro- and macro-systems, as well as within microsystems, for both space and non-space applications. Currently, he is conducting a project resulting in a magnetoresistive magnetometer for 3-D measurement of magnetic fields in space.

Klas Gunnarsson was born in 1962. He received his MSc degree in Engineering Physics in 1986 and his PhD degree in Solid State Physics in 1992, both at Uppsala University. Since 2001, Klas Gunnarsson is employed as a researcher at the department of Engineering Sciences, Uppsala University. His field of research comprises nanomagnetism, biomagnetism, spin dynamics and magnetic materials.

Frederik Westergaard Østerberg obtained his Master of Science degree from Thechical University of Denmark in 2009. Currently, he is a Ph.D student at Thechical University of 
Denmark working on measuring brownian relaxation of magnetic beads using planar Hall effect sensors. His current fields of interests are Hall effect devices and magnetic biosensors.

Bjarke Thomas Dalslet Master of Science, Technical University of Denmark 2004, Dr. Ir. Universiteit Twente 2008. Employed at DTU Nanotech since 2007 he is now an assistant professor. His current fields of interest are micro and nanoscale fabrication and design of magnetic thin film devices and high and low temperature solid state electrochemical cells.

Mikkel Fougt Hansen was born in 1971 and obtained his MSc degree in physics from University of Copenhagen in 1995 and his $\mathrm{PhD}$ degree in physics in 1998 from the Technical University of Denmark. He then pursued post-doctoral positions at Uppsala University and at the Center for Magnetic Recording Research at the University of California, San Diego. Since 2002 he has been associate professor at DTU Nanotech, Department of Micro- and Nanotechnology. His present research interests include magnetic lab-on-a-chip systems, magnetic nanoparticles, magnetic biosensors and applications there of.

Peter Svedlindh was born in 1956 and obtained his MSc degree en engineering physics from Uppsala University in 1980 and his PhD degree in solid state physics in 1985 from the same university. He worked as a researcher at Uppsala University and at the National Defence Research Establishment in Stockholm between 1986-2003, after which he obtained a senior lecturer position at Uppsala University. Since 2000 he is a professor in solid state physics at the Department of Engineering Sciences. His present research interests include spin dynamics, magnetic thin films, magnetic nanoparticles, magnetic biosensors and applications there of.

\section{References}

[1] P. P. Freitas, R. Ferreira, S. Cardoso, F. Cardoso, Magnetoresistive sensors, J. Phys.: Condens. Matter 19 (2007) 165221

[2] Nathan A. Stutzke, Stephan E. Russek, David P. Pappas, Low-frequency noise measurements on commercial magnetoresistive magnetic field measurements, J. Appl. Phys. 97 (2005) 10Q107

[3] R. Ferreira, P. Wisniowski, P. P. Freitas, J. Langer, B. Ocker, W. Maass, Tuning of MgO barrier magnetic tunnel juntion bias current for picotesla magnetic field detection, J. Appl. Phys. 99 (2006) 08K706

[4] R. C. Chaves, P. P. Freitas, B. Ocker, W. Maass, Low frequency picotesla field detection using hybrid MgO based tunnel sensors, Appl. Phys. Lett. 91 (2007) 102504

[5] R. C. Chaves, P. P. Freitas, B. Ocker, W. Maass, MgO based picotesla sensors, J. Appl. Phys. 103 (2008) 07E931

[6] S. H. Liou, D. Sellmyer, S. E. Russek, R. Heindl, F. C. S. Da Silva, J. Moreland, D. P. Pappas, L. Yuan, J. Shen, Magnetic noise in a low-power picotesla magnetoresistive sensor, IEEE SENSORS (2009) 1848

[7] Philip W. T. Pong, Robert McMichaela, Alan S. Edelsteinb, Edmund R. Nowakc, William F. Egelhoff, Jr., Preliminary design and noise considerations for an ultrasensitive magnetic field sensor, Nanoengineering: Fabrication, Properties, Optics, and Devices IV, edited by Elizabeth A. Dobisz, Louay A. Eldada, Proc. of SPIE 6645 (2007) 66450T

[8] H. T. Hardner, M. B. Weissman, B. Miller, R. Loloee, S. S. P. Parkin, Resistance noise in uncoupled giant magnetoresistive multilayers, J. Appl. Phys. 79 (1996) 7751

[9]S. Ingvarsson, Gang Xiao, S. S. P. Parkin, W. J. Gallagher, G. Grinstein, R. H. Koch, Lowfrequency magnetic noise in micron-scale magnetic tunnel junctions, Phys. Rev. Lett. 85 (2000) 3289 
[10] L. Jiang, E. R. Nowak, P. E. Scott, J. Johnson, J. M. Slaughter, J. J. Sun, R. W. Dave, Low-frequency magnetic and resistance noise in magnetic tunnel junctions, Phys. Rev. B 69 (2004) 054407

[11] Cong Ren, Xiaoyong Liu, B. D. Schrag, Gang Xiao, Low-frequency magnetic noise in magnetic tunnel junctions, Phys. Rev. B 69 (2004) 104405

[12] J. M. Almeida, R. Ferreira, P. P. Freitas, J. Langer, B. Ocker, W. Maass, 1/f noise in linearized low resistance MgO magnetic tunnel junctions, J. Appl. Phys. 99 (2006) 08B314 [13] J. H. Scofield, J. V. Mantese, W. W. Webb, 1/f noise of metals: A case for extrinsic origin, Phys. Rev. B 32 (1985) 736

[14] Xiaoyong Liu Gang Xiao, Thermal annealing effects on low-frequency noise and transfer behavior in magnetic tunnel junction sensors, J. Appl. Phys. 94 (2003) 6218

[15] S, H, Liou, Rui Zhang, S. E. Russek, L. Yuan, S. T. Halloran, D. P. Pappas, Dependence of noise in magnetic tunnel junction sensors on annealing field and temperature, J. Appl. Phys. 103 (2008) 07E920

[16] N. Smith, A. M. Zeltser, D. L. Yang, P. V. Koeppe, Very high sensitivity GMR spinvalve magnetometer, IEEE Trans. Magn. 33 (1997) 3385

[17] A. Guedes, J. M. Almeida, S. Cardoso, R. Ferreira, P. P. Freitas, Improving magnetic field detection limits of spin valve sensors using magnetic flux guide concentrators, IEEE Trans. Magn. 43 (2007) 2376

[18] A. S. Edelstein, G. A. Fischer, M. Pedersen, E. R. Nowak, Shu Fan Cheng, C. A. Nordman, Progress toward thousandfold reduction in 1/f noise in magnetic sensors using an ac microelectromechanical system flux concentrator, J. Appl. Phys. 99 (2006) 08B317

[19] L. Ejsing, M. F. Hansen, A. K. Menon, H. A. Ferreira, D. L. Graham, P. P. Freitas, Planar Hall effect sensor for magnetic micro- and nanobead detection, Appl. Phys. Lett. 84 (2004) 4729

[20] B. T. Dalslet, C. D. Damsgaard, M. Donolato, M. Strømme, M. Strömberg, P. Svedlindh, M. F. Hansen, Bead magnetorelaxometry with an on-chip magnetoresistive sensor, Lab Chip $11(2011) 296$

[21] A. D. Henriksen, B. T. Dalslet, D. H. Skieller, K. H. Lee, F. Okkels, M. F. Hansen, Planar Hall effect bridge magnetic field sensors, Appl. Phys. Lett. 97 (2010) 013507

[22] R. Raquet, Electric noise in magnetic materials and devices, Lecture notes in physics 569 (2001) 232-273 doi: 10.1007/3-540-45258-3_11

[23] J. B. Johnson, Thermal Agitation of Electricity in Conductors, Nature 119 (1927) 50 doi:10.1038/119050c0

[24] F. N. Hooge, 1/f noise, Physica B, 83 (1976) 14

[25] C. D. Damsgaard, S. C. Freitas, P. P. Freitas, M. F. Hansen, Exchange-biased planar Hall effect sensors optimized for biosensor applications, J. Appl. Phys. 103 (2008) 07A302

[26] H. Barkhausen, Earth noises and noises due to change of magnetisation of iron, Physikalische Zeitschrift 20 (1919) 401-3

[27] H. T. Hardner, M. B. Weissman M. B. Salamon, Fluctuation-dissipation relation for giant magnetoresistive 1/f noise, Physical Review B 48 (1993) 16156

[28] A. F. Md. Nor and Ernie W. Hill, Noise Power Spectral Density in Single-Strip

NiFeCo-Cu GMR Sensors, IEEE Trans. Mag., 38 (2002) 2697

[29] J. Briaire, L. K. J. Vandamme, M. A. M. Gijs, Resistance and 1/f noise dependence on magnetic field in single Ni80Fe20 layers and Ni80Fe20/Cu multilayers, Journal of Magnetism and Magnetic Materials 165 (1997) 360-362

[30] V. Gehanno, P. P. Freitas, A. Veloso, J. Ferreira, B. Almeida, J. B. Sousa, A. Kling, J. C. Soares, M. F. da Silva, Ion Beam Deposition of Mn-Ir Spin Valves, IEEE Transactions on magnetics, 35 (1999) 4361-7 